\title{
Randomized phase 3 efficacy and safety trial of proposed pegfilgrastim biosimilar MYL-1401H in the prophylactic treatment of chemotherapy-induced neutropenia
}

\author{
Cornelius F. Waller ${ }^{1}$ (D) Gopinath M. Ranganna ${ }^{2} \cdot$ Eduardo J. Pennella $^{3} \cdot$ Christopher Blakeley $^{4} \cdot$ Miguel H. Bronchud $^{5}$. \\ Leonard A. Mattano $\mathrm{Jr}^{6}$ • Oleksandr Berzoy ${ }^{7}$. Nataliia Voitko ${ }^{8}$. Yaroslav Shparyk ${ }^{9}$. Iryna Lytvyn ${ }^{10}$. Andriy Rusyn ${ }^{11}$. \\ Vasil Popov $^{12}$. István Láng ${ }^{13}$. Katrin Beckmann ${ }^{14}$. Rajiv Sharma ${ }^{15}$. Mark Baczkowski ${ }^{16}$ Mudgal Kothekar $^{17}$. \\ Abhijit Barve ${ }^{3}$
}

Received: 26 October 2018 / Accepted: 10 February 2019 / Published online: 1 March 2019

(C) The Author(s) 2019

\begin{abstract}
Pegfilgrastim is indicated for reducing the duration of neutropenia and incidence of febrile neutropenia in patients receiving cytotoxic chemotherapy. Here, safety and efficacy of MYL-1401H, a proposed pegfilgrastim biosimilar, were investigated as prophylaxis for chemotherapy-induced neutropenia. This was a phase 3, multicenter, randomized, double-blind, parallel-group equivalence trial of MYL-1401H vs European Union-sourced reference pegfilgrastim. Patients with newly diagnosed stage II/III breast cancer eligible to receive (neo) adjuvant chemotherapy with docetaxel/doxorubicin/cyclophosphamide every 3 weeks for 6 cycles were enrolled and randomized 2:1 to $6 \mathrm{mg}$ of MYL-1401H or reference pegfilgrastim $24 \mathrm{~h}(+2-\mathrm{h}$ window after the first $24 \mathrm{~h}$ ) after the end of chemotherapy. The primary efficacy endpoint was the duration of severe neutropenia in cycle 1 (i.e., days with absolute neutrophil count $(\mathrm{ANC})<0.5 \times 10^{9} / \mathrm{L}$ ). Mean (standard deviation (SD)) duration of severe neutropenia in MYL$1401 \mathrm{H}$ and reference pegfilgrastim groups was 1.2 days (0.93) and 1.2 days (1.10), respectively. The 95\% CI for least squares mean difference $(-0.285,0.298)$ was within the predefined equivalence range of \pm 1 day. Secondary endpoints, including grade $\geq 3$ neutropenia (frequency, $91 \%$ and $82 \%$ for MYL-1401H and reference pegfilgrastim, respectively), time to ANC nadir (mean (SD), 6.2 (0.98) and 6.3 (1.57) days), and duration of post-nadir recovery (mean (SD), $1.9(0.85)$ and 1.7 (0.91) days) were comparable. Overall safety profiles of the study drugs were comparable. MYL-1401H demonstrated equivalent efficacy and similar safety to reference pegfilgrastim and may be an equivalent option for reducing incidence of neutropenia. (ClinicalTrials. gov, NCT02467868; EudraCT, 2014-002324-27).
\end{abstract}

Keywords Pegfilgrastim $\cdot$ Biosimilar $\cdot$ Febrile neutropenia $\cdot$ Chemotherapy-induced neutropenia

\section{Introduction}

Biologics have great therapeutic promise but are limited by cost and availability [1]. As biologics lose patent protection, biosimilar development may reduce costs and expand access [1-3]. Recently, the European Medicines Agency (EMA) and

Electronic supplementary material The online version of this article (https://doi.org/10.1007/s00277-019-03639-5) contains supplementary material, which is available to authorized users.

Cornelius F. Waller

cornelius.waller@uniklinik-freiburg.de

Extended author information available on the last page of the article the US Food and Drug Administration (FDA) established guidance for biosimilar development and approval [4, 5]. Preclinical characterization, pharmacokinetic/pharmacodynamic (PK/PD), safety, and efficacy studies should be conducted to demonstrate equivalence to reference product $[4,5]$.

Filgrastim, which has been used for febrile neutropenia (FN) prophylaxis in patients receiving myelosuppressive chemotherapy for $>25$ years, was among the first drugs to have biosimilar versions approved by the EMA (2008) and FDA (2015) [6-8]. Filgrastim for chemotherapy-induced neutropenia $(\mathrm{CIN})$ prophylaxis can reduce infection-related mortality in patients with cancer and minimize chemotherapy dose reductions and delays $[9,10]$. Clinical studies have shown that filgrastim biosimilars have pharmacologic, safety, and efficacy profiles comparable to the originator product [11-15]. 
Accordingly, guidelines now indicate filgrastim biosimilars are appropriate for FN prophylaxis in patients receiving myelosuppressive chemotherapy $[15,16]$.

While filgrastim is administered daily, the pegylated form, pegfilgrastim, is only administered once per chemotherapy cycle [16, 17]. Pegfilgrastim was approved in 2002 for CIN prophylaxis, and significant research has gone into pegfilgrastim biosimilar development [3]. Recent preclinical and PK/PD data have supported biosimilarity between originator pegfilgrastim (Neulasta ${ }^{\circledR}$; Amgen Inc., Thousand Oaks, CA) and the proposed biosimilar MYL-1401H [18]. Here, we present data from a phase 3 efficacy and safety trial conducted to confirm equivalence of MYL-1401H with European Union (EU)-sourced reference pegfilgrastim for $\mathrm{CIN}$ prophylaxis.

\section{Materials and methods}

\section{Study design}

This phase 3, randomized, double-blind, parallel-group trial evaluated equivalence of MYL-1401H and reference pegfilgrastim (Neulasta) in patients with breast cancer eligible to receive neoadjuvant or adjuvant TAC (docetaxel $75 \mathrm{mg} / \mathrm{m}^{2}$, doxorubicin $50 \mathrm{mg} / \mathrm{m}^{2}$, cyclophosphamide $500 \mathrm{mg} / \mathrm{m}^{2}$ ) chemotherapy (ClinicalTrials.gov, NCT02467868; EudraCT, 2014-002324-27; Supplementary Fig. 1 in Online Resource). Patients were screened within 4 weeks of chemotherapy initiation, and eligible patients were randomized 2:1 to MYL$1401 \mathrm{H}$ or reference pegfilgrastim via interactive voice/web response system and stratified by age and country. Reference pegfilgrastim (EMA approved) was obtained from European sources.

Randomized patients underwent 6 planned chemotherapy cycles every 3 weeks. In each cycle, chemotherapy was administered on day 1 and a single subcutaneous 6-mg dose of MYL-1401H or reference pegfilgrastim was administered $24 \mathrm{~h}$ (+2-h window after the first $24 \mathrm{~h}$ ) after the end of chemotherapy. Only the pharmacist preparing the doses and the person (not the principal investigator) administering treatment were unblinded.

All patients were aged $\geq 18$ years with newly diagnosed stage II/III breast cancer and adequate staging workup and surgery if receiving adjuvant therapy. Patients were required to be chemotherapy and radiotherapy naive with Eastern Cooperative Oncology Group performance status $\leq 1$ and absolute neutrophil count (ANC) $\geq 1.5 \times 10^{9} / \mathrm{L}$ at baseline. Patients previously exposed to filgrastim products were excluded as they can potentially develop antidrug antibodies (ADA) that may interfere with study assessments [14].

The choices of patient population and chemotherapy regimen were determined using guidance from regulatory agencies. As recommendations support routine use of filgrastim in patients receiving chemotherapy regimens that are associated with a $>20 \%$ risk of FN, prophylactic treatment with pegfilgrastim in this patient population is consistent with current guidelines $[15,16]$.

The clinical study protocol, informed consent forms, and all other appropriate study-related documents were approved by local independent ethics committees and institutional review boards, as applicable. The study was conducted in accordance with International Council for Harmonization Guideline for Good Clinical Practice and the Declaration of Helsinki at 25 sites in Bulgaria, Georgia, Hungary, and Ukraine between March 25, 2015, and February 9, 2016.

\section{Endpoints}

The primary efficacy endpoint was the duration of severe neutropenia (DSN) in cycle 1, defined as days with $\mathrm{ANC}<0.5 \times$ $10^{9} / \mathrm{L}$. During cycle 1 , ANC was determined from blood samples collected daily for 15 days after chemotherapy administration. In subsequent cycles, ANC was measured on days 8 , 11 , and 15 .

Measurements of ANC were used to calculate secondary efficacy endpoints: frequency of grade 3 or 4 neutropenia, depth and time to ANC nadir and rate of recovery, and rate of FN. Febrile neutropenia was defined per European Society for Medical Oncology as ANC $<0.5 \times 10^{9} / \mathrm{L}$ or expected to fall below $0.5 \times 10^{9} / \mathrm{L}$, with a single oral temperature $>$ $38.5^{\circ} \mathrm{C}$ or 2 consecutive readings $>38.0^{\circ} \mathrm{C}$ for $2 \mathrm{~h}$. Delay, reduction, and omission of chemotherapy doses were also recorded.

All adverse events (AEs), regardless of relationship to study drug, were recorded through 28 days after the last administration of MYL-1401H or reference pegfilgrastim. Adverse events were graded using the National Cancer Institute Common Terminology Criteria for Adverse Events (version 4.03). In the first 2 cycles, bone pain was measured daily for the first 15 days by the Brief Pain Inventory (BPI) short form [19]. Injection site reactions were documented during all cycles. Blood samples for assessment of ADA and neutralizing antibodies (NAb) were collected on day 1, cycle 1 , and at the end of cycles 2, 4, and 6 .

\section{Statistical analysis}

A sample size of 135 patients randomized 2:1 (90 and 45 patients treated with MYL-1401H and reference pegfilgrastim, respectively) was required to provide $90 \%$ power to declare equivalence between MYL-1401H and reference pegfilgrastim for analysis of DSN in cycle 1. This sample size assumed that the mean DSN was 1.7 days in cycle 1 for both MYL-1401H and reference pegfilgrastim, with a common standard deviation (SD) of 1.5 days [20,21], and the difference between mean DSN was analyzed with a 2-sided $95 \%$ CI. The required sample 
size of 135 patients was increased to 189 to allow for attrition and ensure that safety was assessed in $\sim 100$ patients receiving MYL-1401H for 6 cycles per EMA requirement.

Primary efficacy analysis was conducted in the per protocol (PP) population (randomized patients without major protocol deviations who received $\geq 1$ study drug dose). Analysis of variance (ANOVA) model with treatment as an independent factor was used to produce a 2-sided $95 \%$ CI for difference in the least square mean DSN. Equivalence was declared if the CI was completely within the range of \pm 1 day. The equivalence margin was in alignment with guidelines provided by regulatory authorities (FDA and EMA) [22]. Secondary efficacy endpoints were analyzed descriptively. Chi-square test was used to assess the difference in the occurrence of FN between treatment groups. Safety was analyzed in patients who received $\geq 1$ study drug dose (safety population). The intention-to-treat (ITT) population included all randomized patients. All statistical analyses were performed using SAS® (Cary, NC).

\section{Results}

\section{Patient disposition}

Of 207 patients screened, 194 were randomized, 127 received MYL-1401H, and 67 received reference pegfilgrastim (Supplementary Fig. 2 in Online Resource). All randomized patients completed cycle 1 and were included in both the ITT and safety populations. One patient in the MYL-1401H group took a prohibited concomitant medication and was excluded from the PP population. All 6 chemotherapy cycles were completed by 120 patients in the MYL- $1401 \mathrm{H}$ group and 66 in the reference pegfilgrastim group.

\section{Baseline characteristics}

No differences were observed in baseline characteristics between groups (Table 1). Median age (range) was 49 years (2579 ) in the MYL-1401H group and 50 years (29-68) in the reference pegfilgrastim group. All $67(100 \%)$ patients in the reference pegfilgrastim group and 126 (99\%) in the MYL$1401 \mathrm{H}$ group were female. All 194 patients were white.

\section{Efficacy}

\section{Primary endpoint}

In the PP population, mean (SD) DSN for MYL-1401H was 1.2 days (0.93), and median (range) DSN was 1.0 day (0-5; Table 2). Mean (SD) DSN for reference pegfilgrastim was 1.2 days (1.10), and median (range) DSN was 1.0 day (0-4). The $95 \%$ CI $(-0.285,0.298)$ for the difference in LS mean
DSN of MYL-1401H and reference pegfilgrastim was entirely within the prespecified equivalence range of \pm 1 day based on the ANOVA model. Therefore, equivalent efficacy of MYL$1401 \mathrm{H}$ and reference pegfilgrastim was concluded.

\section{Secondary endpoints}

Mean ANC profiles of MYL-1401H- and reference pegfilgrastim-treated patients were similar in cycle 1 (PP population; Fig. 1). Mean (SD) time to ANC nadir was 6.2 days (0.98) and 6.3 days (1.57) for MYL-1401H and reference pegfilgrastim, respectively. Median (range) ANC nadir was $0.21 \times 10^{9} / \mathrm{L}\left(0.0-2.5 \times 10^{9} / \mathrm{L}\right)$ and $0.27 \times 10^{9} / \mathrm{L}(0.0$ $6.7 \times 10^{9} / \mathrm{L}$ ) for MYL-1401H and reference pegfilgrastim, respectively. All patients in cycle 1 receiving reference pegfilgrastim had post-nadir ANC recovery. Post-nadir ANC recovery could not be established in 1 patient $(1 \%)$ receiving MYL-1401H because the patient did not have 2 recovery observations before the end of cycle 1 . However, all evaluable patients (i.e., those with 2 recovery observations) receiving MYL-1401H had post-nadir ANC recovery. In patients with confirmed post-nadir ANC recovery, mean (SD) time to recovery was 1.9 days $(0.85)$ and 1.7 days $(0.91)$ for MYL-1401H and reference pegfilgrastim, respectively. Overall, $97 \%$ of patients $(121 / 125)$ receiving MYL-1401H and all patients receiving reference pegfilgrastim with a confirmed post-nadir ANC recovery in cycle 1 had recovery time of $\leq 3$ days.

In cycle 1 , grade $\geq 3$ neutropenia occurred in $91 \%$ of patients (114/126) receiving MYL-1401H and 82\% (55/67) receiving reference pegfilgrastim; grade 4 neutropenia occurred in $75 \%$ of patients $(94 / 126)$ receiving MYL-1401H and $64 \%$ (43/67) receiving reference pegfilgrastim (PP population). However, in a majority of patients, grade 4 neutropenia did not recur in all cycles. Overall, in $19 \%$ of patients receiving MYL-1401H and $27 \%$ receiving reference pegfilgrastim, no grade 4 neutropenia occurred. Sixty-one patients $(48 \%)$ receiving MYL-1401H and 31 (46\%) receiving reference pegfilgrastim had grade 4 neutropenia in 1 or 2 cycles only. Less than $10 \%$ of patients had grade 4 neutropenia in all 6 cycles: $7 \%$ and 9\% receiving MYL-1401H and reference pegfilgrastim, respectively. No prolonged neutropenia ( $>10$ days) was reported, and profound neutropenia $(<0.1 \times$ $\left.10^{9} / \mathrm{L}\right)$ incidence was similar between groups. Across all cycles, FN occurred in $6 \%$ of patients (7/127) receiving MYL$1401 \mathrm{H}$ and $2 \%(1 / 67)$ receiving reference pegfilgrastim (ITT population). Most FN events occurred during cycle 1: MYL$1401 \mathrm{H}, 4 \%(5 / 127)$ and reference pegfilgrastim, 2\% (1/67). Although there were numerical differences in $\mathrm{FN}$ rates, these differences were not significant for cycle 1 (difference, 2.4; $95 \% \mathrm{CI},-2.0,6.9 ; P=0.35$ ) or 6 cycles (difference, $4.0 ; 95 \%$ $\mathrm{CI},-0.9,8.9 ; P=0.18)$. Post hoc analysis showed that MYL$1401 \mathrm{H}$ is noninferior to reference pegfilgrastim for FN 
Table 1 Patient Characteristics (ITT Population)

\begin{tabular}{lccc}
\hline Parameter & MYL-1401H $(N=127)$ & Reference $(N=67)$ & Overall $(N=194)$ \\
\hline Age, $y$ & & & \\
Mean (SD) & $50(11)$ & $50(10)$ & $50(10)$ \\
Median (range) & $49(25-79)$ & $50(29-68)$ & $50(25-79)$ \\
Age group, y, $n(\%)$ & & & \\
$<50$ & $64(50)$ & $32(48)$ & $96(50)$ \\
$50-65$ & $56(44)$ & $30(45)$ & $86(44)$ \\
$>65$ & $7(6)$ & $5(8)$ & $12(6)$ \\
White, $n(\%)$ & $127(100)$ & $67(100)$ & $194(100)$ \\
Sex, $n(\%)$ & & & $193(1)$ \\
Female & $126(99)$ & $67(100)$ & $49(25)$ \\
Male & $1(1)$ & $0(0)$ & $64(33)$ \\
TNM stage at diagnosis, $n(\%)$ & $15(22)$ & $42(22)$ \\
IIa & $34(27)$ & $22(33)$ & $13(7)$ \\
IIb & $42(33)$ & $16(24)$ & $26(13)$ \\
IIIla & $26(21)$ & $7(10)$ & $78(40)$ \\
IIIlb & $6(5)$ & $7(10)$ & \\
IIIc & $19(15)$ & $43(64)$ & \\
Indicated treatment, $n(\%)$ & & $24(36)$ & \\
Adjuvant & $73(58)$ & & \\
Neoadjuvant & $54(42)$ & & \\
\hline
\end{tabular}

ITT intention-to-treat, $S D$ standard deviation, TNM tumor node metastasis staging system incidence, assuming a noninferiority margin of 9\%. All FN incidences were of short duration and resolved within 5 days of onset; no infections required treatment or rescue therapy with filgrastim, and no infection-related mortality was observed. Reductions, omissions, or delays in chemotherapy related to neutropenia, $\mathrm{FN}$, or documented infections occurred in $4 \%$ of patients $(5 / 127)$ receiving MYL-1401H and $2 \%$ (1/67) receiving reference pegfilgrastim (ITT population).

The isolated differences between groups in incidence of grade $\geq 3$ neutropenia; occurrence of FN; and reductions, omissions, or delays in chemotherapy were observed in exploratory analyses not powered for comparison. No significant infections or sepsis were reported in either group during FN or grade $\geq 3$ neutropenia events.

\section{Safety}

Similar rates of treatment-emergent AEs (TEAEs) were observed between groups (Table 3). A total of 806 TEAEs were reported in $114(90 \%)$ patients receiving MYL-1401H, and 414 TEAEs were reported in $58(87 \%)$ patients receiving reference pegfilgrastim. One patient receiving MYL-1401H experienced a grade $\geq 4$ TEAE (grade $4 \mathrm{FN}$ ), which was considered unrelated to study drug. No patients receiving reference pegfilgrastim experienced any grade $\geq 4$ TEAEs. No MYL$1401 \mathrm{H}$-treated patients reported an injection site reaction. One $(2 \%)$ patient receiving reference pegfilgrastim had injection site redness on day 2 , cycle 2 , and 1 (2\%) had bruising on day 8 , cycle 2 and day 8 , cycle 3 . No deaths, treatmentrelated discontinuations, or suspected unexpected serious adverse reactions occurred in either group.

For both groups, mean highest pain score, assessed by averaging individual patient responses to question 3 (i.e., worst pain in the last $24 \mathrm{~h}$ ) of the BPI in cycle 1 (Supplementary Fig. 3 in Online Resource), was reported on day 4 , followed by plateauing and eventual decline by day 15 . Bone pain reported by patients was similar between groups, and no patients discontinued because of bone pain. However, naproxen use for bone pain management in cycle 1 was higher with reference pegfilgrastim (19 (28\%)) than MYL-1401H (25 (20\%)).

Of the patients in the safety population with available baseline immunogenicity results, $15 \%$ (19/126) receiving MYL$1401 \mathrm{H}$ and $18 \%(12 / 67)$ receiving reference pegfilgrastim were ADA positive before dosing. Of those with available immunogenicity results after treatment initiation, $1 \%(1 / 125)$ receiving MYL-1401H and 3\% (2/67) receiving reference pegfilgrastim were ADA positive. Most ADA-positive samples before treatment were against the polyethylene glycol moiety. No ADA-positive patients were Nab positive. Only 1 (2\%) ADA-positive result for reference pegfilgrastim was due to seroconversion, defined as ADA negative at baseline but with subsequent positive response. The MYL-1401H group had no treatment-induced ADA-positive patients. 
Table 2 Efficacy Endpoints

\begin{tabular}{|c|c|c|}
\hline $\begin{array}{l}\text { Frequency, depth, and time of neutropenia } \\
\text { in cycle } 1 \text { (PP population) }\end{array}$ & MYL-1401H $(N=126)$ & Reference $(N=67)$ \\
\hline DSN, mean (SD), days & $1.2(0.9)$ & $1.2(1.1)$ \\
\hline DSN, LS mean (SE), days & $1.3(0.1)$ & $1.3(0.2)$ \\
\hline Grade 3 neutropenia, $n(\%)^{\mathrm{a}, \mathrm{b}}$ & $20(15.9)$ & $12(17.9)$ \\
\hline Grade 4 neutropenia, $n(\%)^{\mathrm{b}, \mathrm{c}}$ & $94(74.6)$ & $43(64.2)$ \\
\hline ANC nadir, mean $(\mathrm{SD}), 10^{9} / \mathrm{L}$ & $0.40(0.5)$ & $0.78(1.4)$ \\
\hline ANC nadir, median (range), $10^{9} / \mathrm{L}$ & $0.21(0.0-2.5)$ & $0.27(0.0-6.7)$ \\
\hline Duration of post-nadir ANC recovery within $\leq 3$ days, $n(\%)$ & $121(97)^{\mathrm{d}}$ & $67(100)$ \\
\hline Rate of febrile neutropenia in cycle 1 (ITT population) & MYL-1401H $(N=127)$ & Reference $(N=67)$ \\
\hline Rate of febrile neutropenia, $\mathrm{n}(\%)$ & $5(4)$ & $1(2)$ \\
\hline Frequency of neutropenia across all cycles (ITT population) & MYL-1401H $(N=127)$ & Reference $(N=67)$ \\
\hline Grade 3 neutropenia, $n(\%)^{\mathrm{a}, \mathrm{b}}$ & $17(13)$ & $7(10)$ \\
\hline Grade 4 neutropenia, $n(\%)^{\mathrm{b}, \mathrm{c}}$ & $103(81)$ & $49(73)$ \\
\hline Febrile neutropenia, $n(\%)^{\mathrm{e}}$ & $7(6)$ & $1(2)$ \\
\hline $\begin{array}{l}\text { Frequency of chemotherapy doses reduced, omitted, } \\
\text { or delayed across all cycles (ITT population) }\end{array}$ & MYL-1401H $(N=127)$ & Reference $(N=67)$ \\
\hline $\begin{array}{l}\text { Related to neutropenia, febrile neutropenia, } \\
\text { or documented infections, } n(\%)\end{array}$ & $5(4)$ & $1(2)$ \\
\hline
\end{tabular}

$A N C$ absolute neutrophil count, DSN duration of severe neutropenia, ESMO European Society for Medical Oncology, ITT intention-to-treat, $L S$ least squares, $P P$ per protocol, $S D$ standard deviation, $S E$ standard error

${ }^{\text {a }} \mathrm{ANC}<1.0 \times 10^{9} / \mathrm{L}$.

${ }^{\mathrm{b}}$ Only the highest grade neutropenia experienced was reported

${ }^{\mathrm{c}} \mathrm{ANC}<0.5 \times 10^{9} / \mathrm{L}$.

${ }^{\mathrm{d}}$ Percentage calculated using the 125 patients with a confirmed post-nadir ANC recovery

${ }^{\mathrm{e}}$ Out of 8 patients with febrile neutropenia, 3 met the ESMO definition, 1 patient did not, and 4 other patients had insufficient data to confirm febrile neutropenia, but all patients were considered to have febrile neutropenia for the data analysis

\section{Discussion}

The study met the primary endpoint as MYL-1401H efficacy was equivalent for reference pegfilgrastim. The DSN was equivalent for MYL-1401H and reference pegfilgrastim, and mean DSN in cycle 1 was similar to other studies of both biosimilar and reference pegfilgrastim [20,21, 23]. Profiles for ANC were similar between groups throughout all chemotherapy cycles. Robust and correlative PK/PD studies are sensitive at detecting subtle differences in biosimilar efficacy. Previous PK/PD analysis of MYL-1401H demonstrated equivalence with reference pegfilgrastim [18], consistent with efficacy data from this study.

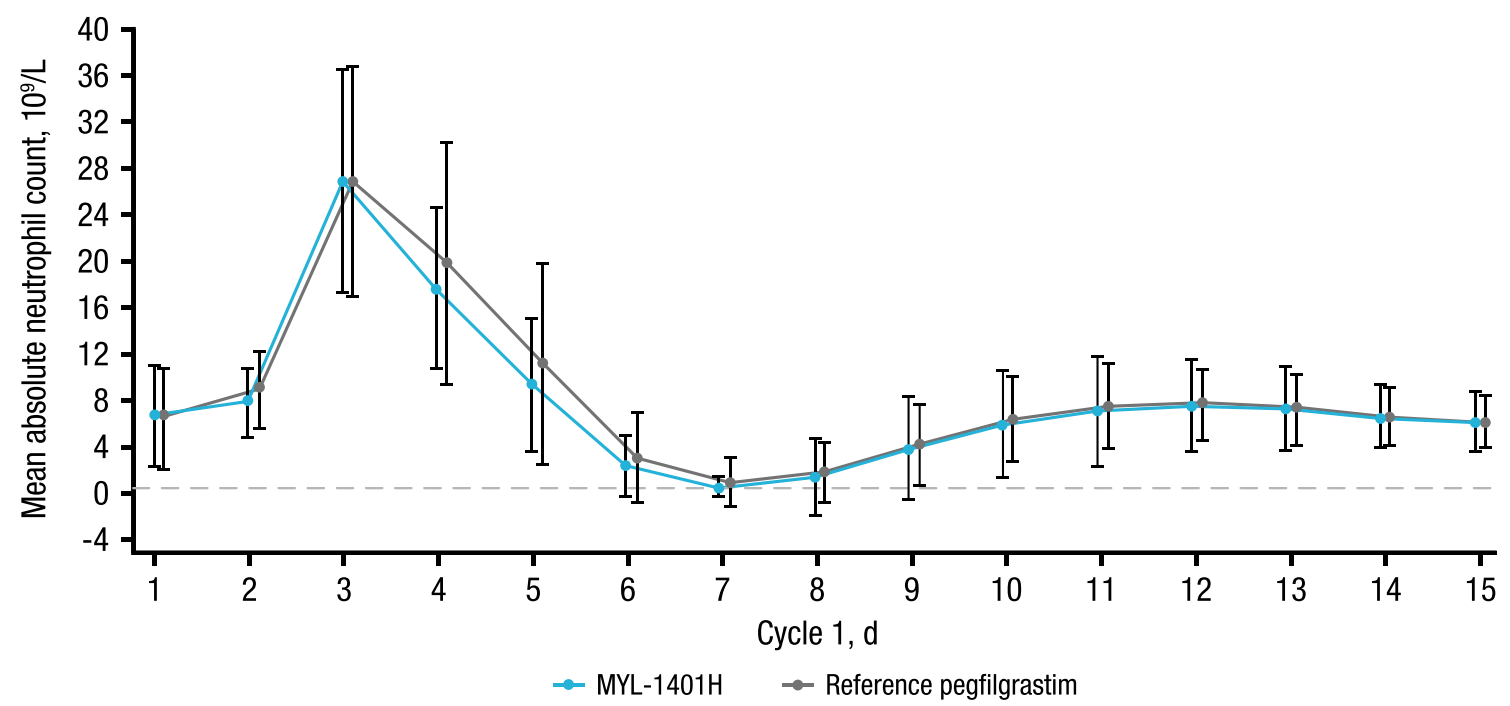

Fig. 1 Mean ANC over time by treatment in cycle 1 (PP population). ANC absolute neutrophil count, $P P$ per protocol 
A slightly higher incidence of grade 4 neutropenia occurred with MYL-1401H (75\%) vs reference pegfilgrastim (64\%). However, grade 4 neutropenia recurred across all cycles in only $7 \%$ and $9 \%$ of patients receiving MYL-1401H and reference pegfilgrastim, respectively. The majority of grade 4 neutropenia events lasted $<2$ days in both groups. The incidence of FN was slightly higher in the MYL-1401H treatment group; however, the difference was not statistically significant. There were no documented infections associated with grade $4 \mathrm{FN}$, and few patients (MYL-1401H, 5 (4\%); reference pegfilgrastim, $1(2 \%)$ ) had their chemotherapy dose modified. Historical data for pegfilgrastim from other studies with similar populations and chemotherapy regimens have shown that incidence of $\mathrm{FN}$ with reference pegfilgrastim was up to $10 \%$ in cycle 1 and $13 \%$ in all cycles, with a low mean depth of ANC nadir [23, 24], similar to the range seen in patients receiving MYL-1401H. For example, mean depth of ANC nadir was $0.49 \times 10^{9} / \mathrm{L}(\mathrm{SD}, 0.72$; median, 0.24; range, 0.0 4.4) and $0.44 \times 10^{9} / \mathrm{L}$ (SD, 0.57; median, 0.30; range, 0.0-3.8) in patients receiving LA-EP2006 or reference pegfilgrastim, respectively [23]. Therefore, isolated differences in grade 4 neutropenia, FN, and ANC nadir between treatment groups were not considered clinically significant and possibly due to sample size limitations.

Both products were generally well tolerated, and only minor differences in TEAE rates and secondary efficacy endpoints were observed, which were not clinically meaningful. No unexpected serious adverse reactions occurred. One of the most frequently reported treatment-related TEAEs was bone pain, and incidence of bone pain was similar to that reported in other studies of pegfilgrastim in patients receiving myelosuppressive chemotherapy other than TAC (range, 25-38\%) [25]. Rare, serious side effects of G-CSF therapy [26], such as splenomegaly, acute respiratory distress syndrome, capillary leak syndrome, and severe allergic reactions, were not observed in this study. There are limitations when comparing these results with those from similar studies because of varying patient populations, chemotherapy regimens, and other factors related to study design. On balance, the efficacy and safety results from this study are in accordance with published literature on biosimilar G-CSF products [11-14, 18, 23, 24].

\section{Conclusions}

This study demonstrated that MYL- $1401 \mathrm{H}$ is equivalent in efficacy to originator pegfilgrastim for prophylaxis of CIN in patients with breast cancer treated with neoadjuvant or adjuvant TAC chemotherapy, with no clinically meaningful differences in safety. Given the equivalence between groups regarding primary and secondary endpoints and known clinical benefits of G-CSF, these results can be applied to many chemotherapy regimens that result in high rates of $\mathrm{FN}$, independent of tumor type.
Table 3 TEAEs by Preferred Term Across All Cycles (Safety Population)

\begin{tabular}{lcc}
\hline & MYL-1401H $(N=127)$ & Reference $(N=67)$ \\
\hline TEAEs, $n$ & 806 & 414 \\
Patients with TEAEs, $n(\%)$ & $114(90)$ & $58(87)$ \\
Patients with TEAEs grade $\geq 4, n(\%)$ & $1(1)^{\mathrm{a}}$ & $0(0)$ \\
TEAEs occurring in $\geq 5 \%$ patients in either treatment group, $n(\%)$ & $36(54)$ \\
Alopecia & $76(60)$ & $24(36)$ \\
Bone pain & $51(40)$ & $25(37)$ \\
Nausea & $37(29)$ & $10(15)$ \\
Asthenia & $23(18)$ & $16(24)$ \\
Fatigue & $19(15)$ & $12(18)$ \\
Diarrhea & $16(13)$ & $6(9)$ \\
Thrombocytopenia & $14(11)$ & $9(13)$ \\
Anemia & $14(11)$ & $7(10)$ \\
Vomiting & $12(9)$ & $8(12)$ \\
Headache & $12(9)$ & $2(3)$ \\
Stomatitis & $11(9)$ & $0(0)$ \\
Thrombocytosis & $8(6)$ & $0(0)$ \\
Decreased appetite & $8(6)$ & $1(1)$ \\
Febrile neutropenia & $7(6)$ & $8(12)$ \\
Alanine aminotransferase increased & $7(6)$ & $7(10)$ \\
Aspartate aminotransferase increased & $7(6)$ & $5(7)$ \\
Platelet count decreased & $7(6)$ & $5(7)$ \\
Pyrexia & $3(2)$ & $4(6)$ \\
Abdominal pain & $3(2)$ & \\
\hline
\end{tabular}

TEAE, treatment-emergent adverse event

${ }^{a}$ Grade 4 febrile neutropenia 
Acknowledgment Editorial assistance was provided under the direction of the authors by Scott Houck, PhD, Rebecca E. Slager, PhD, and Jennifer Rossi, MA, ELS, MedThink SciCom, with support from Mylan Inc.

Funding information This study was funded by Mylan Inc., Canonsburg, PA, and Biocon Limited, Bangalore, India.

Data availability Anonymized individual participant data and study documents can be requested for further research from Joseph Capasso (Joseph.Capasso@mylan.com).

\section{Compliance with ethical standards}

Conflict of interest CF Waller and MH Bronchud are consultants/ advisory board members for Mylan. GM Ranganna, EJ Pennella, K Beckmann, M Baczkowski, and A Barve are paid employees of Mylan and may hold stock with the company. C Blakeley is a paid employee of Worldwide Clinical Trials. LA Mattano Jr. has served as a consultant for Alexion, Mylan, Novartis, and Pfizer and may hold stock with Pfizer. R Sharma was a paid employee of Mylan at the time of analysis and may hold stock with the company. M Kothekar is a paid employee of Biocon Research Ltd. and may hold stock with the company. All remaining authors declare that they have no conflict of interest.

Ethical approval All procedures performed in studies involving human participants were in accordance with the ethical standards of the institutional and/or national research committee and with the 1964 Helsinki declaration and its later amendments or comparable ethical standards.

Statement of informed consent Informed consent was obtained from all individual participants included in the study.

Open Access This article is distributed under the terms of the Creative Commons Attribution 4.0 International License (http:// creativecommons.org/licenses/by/4.0/), which permits unrestricted use, distribution, and reproduction in any medium, provided you give appropriate credit to the original author(s) and the source, provide a link to the Creative Commons license, and indicate if changes were made.

Publisher's note Springer Nature remains neutral with regard to jurisdictional claims in published maps and institutional affiliations.

\section{References}

1. Blackstone EA, Joseph PF (2013) The economics of biosimilars. Am Health Drug Benefits 6:469-478

2. Mellstedt H, Niederwieser D, Ludwig H (2008) The challenge of biosimilars. Ann Oncol 19:411-419. https://doi.org/10.1093/ annonc/mdm345

3. Chopra R, Lopes G (2017) Improving access to cancer treatments: the role of biosimilars. J Glob Oncol 2016:008607. https://doi.org/ 10.1200/JGO.2016.008607

4. US Food and Drug Administration (2015) Scientific considerations in demonstrating biosimilarity to a reference product: guidance for industry. https://www.fda.gov/downloads/Drugs/ GuidanceComplianceRegulatoryInformation/Guidances/ UCM291128.pdf. Accessed 16 August 2018

5. European Medicines Agency (2014) Guideline on similar biological medicinal products containing biotechnology-derived proteins as active substance: non-clinical and clinical issues. http://www.
ema.europa.eu/docs/en_GB/document_library/Scientific guideline/2015/01/WC500180219.pdf. Accessed 16 August 2018

6. Raedler LA (2016) Zarxio (filgrastim-sndz): first biosimilar approved in the United States. Am Health Drug Benefits 9:150-154

7. NEUPOGEN (2016) prescribing information. Amgen Inc, Thousand Oaks

8. Minghetti P, Rocco P, Cilurzo F, Vecchio LD, Locatelli F (2012) The regulatory framework of biosimilars in the European Union. Drug Discov Today 17:63-70. https://doi.org/10.1016/j.drudis.2011.08.001

9. Kuderer NM (2011) Meta-analysis of randomized controlled trials of granulocyte colony-stimulating factor prophylaxis in adult cancer patients receiving chemotherapy. Cancer Treat Res 157:127143. https://doi.org/10.1007/978-1-4419-7073-2_8

10. Dale DC (2002) Colony-stimulating factors for the management of neutropenia in cancer patients. Drugs 62(suppl 1):1-15

11. Blackwell K, Semiglazov V, Krasnozhon D, Davidenko I, Nelyubina L, Nakov R, Stiegler G, Singh P, Schwebig A, Kramer S, Harbeck N (2015) Comparison of EP2006, a filgrastim biosimilar, to the reference: a phase III, randomized, double-blind clinical study in the prevention of severe neutropenia in patients with breast cancer receiving myelosuppressive chemotherapy. Ann Oncol 26:1948-1953. https://doi.org/10.1093/annonc/mdv281

12. del Giglio A, Eniu A, Ganea-Motan D, Topuzov E, Lubenau H (2008) XM02 is superior to placebo and equivalent to Neupogen in reducing the duration of severe neutropenia and the incidence of febrile neutropenia in cycle 1 in breast cancer patients receiving docetaxel/doxorubicin chemotherapy. BMC Cancer 8:332. https:// doi.org/10.1186/1471-2407-8-332

13. Gascon P, Fuhr U, Sörgel F, Kinzig-Schippers M, Makhson A, Balser S, Einmahi S, Muenzberg M (2010) Development of a new G-CSF product based on biosimilarity assessment. Ann Oncol 21:1419-1429. https://doi.org/10.1093/annonc/mdp574

14. Waller CF, Semiglazov VF, Tjulandin S, Bentsion D, Chan S, Challand R (2010) A phase III randomized equivalence study of biosimilar filgrastim versus Amgen filgrastim in patients receiving myelosuppressive chemotherapy for breast cancer [erratum appears in Onkologie 2010;33(12):725]. Onkologie 33:504-511. https:// doi.org/10.1159/000319693

15. Klastersky J, de Naurois J, Rolston K, Rapoport B, Maschmeyer G, Aapro M, Herrstedt J, for ESMO Guidelines Committee (2016) Management of febrile neutropaenia: ESMO clinical practice guidelines. Ann Oncol 27:v111-v118. https://doi.org/10.1093/ annonc/mdw325

16. Smith TJ, Bohlke K, Lyman GH, Carson KR, Crawford J, Cross SJ, Goldberg JM, Khatcheressian JL, Leighl NB, Perkins CL, Somlo G, Wade JL, Wozniak AJ, Armitage JO (2015) Recommendations for the use of WBC growth factors: American Society of Clinical Oncology clinical practice guideline update. J Clin Oncol 33:3199-3212

17. Holmes FA, Jones SE, O'Shaughnessy J, Vukelja S, George T, Savin M, Richards D, Glaspy J, Meza L, Cohen G, Dhami M, Budman DR, Hackett J, Brassard M, Yang BB, Liang BC (2002) Comparable efficacy and safety profiles of once-per-cycle pegfilgrastim and daily injection filgrastim in chemotherapyinduced neutropenia: a multicenter dose-finding study in women with breast cancer. Ann Oncol 13:903-909

18. Waller CF, Tiessen RG, Lawrence TE, Shaw A, Liu MS, Sharma R, Baczkowski M, Kothekar MA, Micales CE, Barve A, Ranganna GM, Pennella EJ (2018) A pharmacokinetics and pharmacodynamics equivalence trial of the proposed pegfilgrastim biosimilar, MYL-1401H, versus reference pegfilgrastim. J Cancer Res Clin Oncol 144:1087-1095. https://doi.org/10.1007/s00432-018-2643-3

19. Cleeland CS (2009) The Brief Pain Inventory. https://www. mdanderson.org/documents/Departments-and-Divisions/SymptomResearch/BPI_UserGuide.pdf. Accessed 16 August 2018

20. Holmes FA, O'Shaughnessy JA, Vukelja S, Jones SE, Shogan J, Savin M, Glaspy J, Moore M, Meza L, Wiznitzer I, Neumann 
TA, Hill LR, Liang BC (2002) Blinded, randomized, multicenter study to evaluate single administration pegfilgrastim once per cycle versus daily filgrastim as an adjunct to chemotherapy in patients with high-risk stage II or stage III/IV breast cancer. J Clin Oncol 20: 727-731. https://doi.org/10.1200/JCO.2002.20.3.727

21. Green MD, Koelbl H, Baselga J, Galid A, Guillem V, Gascon P, Siena S, Lalisang RI, Samonigg H, Clemens MR, Zani V, Liang BC, Renwick J, Piccart M, for the International Pegfilgrastim 749 Study Group (2003) A randomized double-blind multicenter phase III study of fixed-dose single-administration pegfilgrastim versus daily filgrastim in patients receiving myelosuppressive chemotherapy. Ann Oncol 14:29-35

22. European Medicines Agency (2005) Guideline on the choice of the non-inferiority margin. http://www.ema.europa.eu/docs/en_GB/ document_library/Scientific_guideline/2009/09/WC500003636. pdf. Accessed 16 August 2018

23. Blackwell K, Donskih R, Jones CM, Nixon A, Vidal MJ, Nakov R, Singh P, Schaffar G, Gascón P, Harbeck N (2016) A comparison of proposed biosimilar LA-EP2006 and reference pegfilgrastim for the prevention of neutropenia in patients with early-stage breast cancer receiving myelosuppressive adjuvant or neoadjuvant chemotherapy: pegfilgrastim randomized oncology (supportive care) trial to evaluate comparative treatment (PROTECT-2), a phase III, randomized, double-blind trial. Oncologist 21:789-794. https://doi. org/10.1634/theoncologist.2016-0011

24. Harbeck N, Lipatov O, Frolova M, Udovitsa D, Topuzov E, GaneaMotan DE, Nakov R, Singh P, Rudy A, Blackwell K (2016) Randomized, double-blind study comparing proposed biosimilar LA-EP2006 with reference pegfilgrastim in breast cancer. Future Oncol 12:1359-1367. https://doi.org/10.2217/fon-2016-0016

25. Lambertini M, Del Mastro L, Bellodi A, Pronzato P (2014) The five "Ws" for bone pain due to the administration of granulocyte-colony stimulating factors (G-CSFs). Crit Rev Oncol Hematol 89:112-128. https://doi.org/10.1016/j.critrevonc.2013.08.006

26. Neulasta (2016) prescribing information. Amgen Inc, Thousand Oaks

\section{Affiliations}

Cornelius F. Waller ${ }^{1}$ (D) $\cdot$ Gopinath M. Ranganna ${ }^{2} \cdot$ Eduardo J. Pennella $^{3} \cdot$ Christopher Blakeley $^{4} \cdot$ Miguel H. Bronchud $^{5}$. Leonard A. Mattano $\mathrm{Jr}^{6}$. Oleksandr Berzoy ${ }^{7}$. Nataliia Voitko ${ }^{8}$. Yaroslav Shparyk ${ }^{9}$. Iryna Lytvyn ${ }^{10}$. Andriy Rusyn ${ }^{11}$. Vasil Popov ${ }^{12} \cdot$ István Láng $^{13} \cdot$ Katrin Beckmann ${ }^{14} \cdot$ Rajiv Sharma $^{15} \cdot$ Mark Baczkowski $^{16} \cdot$ Mudgal Kothekar $^{17}$. Abhijit Barve $^{3}$

1 Department of Haematology, Oncology and Stem Cell Transplantation, University Medical Centre Freiburg and Faculty of Medicine, University of Freiburg, Hugstetter Street 55, 79106 Freiburg, Germany

2 Global Clinical Research \& Development, Mylan, Prestige Tech Park Platina-3, 7th to 12th Floor, Kadubesanahalli,

Bangalore 560103, India

3 Global Clinical Research, Mylan, 1000 Mylan Boulevard, Canonsburg, PA 15317, USA

4 Medical and Scientific Affairs, Worldwide Clinical Trials, 172 Tottenham Court Road, Fitzrovia, London W1T 7DL, UK

5 GenesisCare Corachan Institute of Oncology, Buïgas 19, 08017 Barcelona, Spain

6 HARP Pharma Consulting, LLC, 184 Masons Island Road, Mystic, CT 06355, USA

7 Mammalogy Center, Odessa Regional Hospital, Akademika Zabolotnogo Str. 26, Odessa 65025, Ukraine

8 Chemotherapy II, Kyiv City Clinical Oncological Centre, Kyiv, Street, Verkhovyna, 69, Kiev 03115, Ukraine

9 Department of Chemotherapy, Lviv State Regional Treatment and Diagnostics Oncology Center, Lviv Street, Pekarskaya, 69,

Lviv 79010, Ukraine
10 Department of Chemotherapy, Dnipropetrovsk Regional Clinical Oncology Center, Kosmicheskaja Street, 21, Dnepropetrovsk 49100, Ukraine

11 Department of Chemotherapy, Transkarpathian Regional University Oncology Clinic, Narodna Square, 3, Uzhgorod 88000, Ukraine

12 Department of Medical Oncology and Palliative Care, SHATOD Dr. Marko Antonov Markov, Varna EOOD, SHOP "Tsar Osvoboditel” Boulevard 100000, 9000 Varna, Bulgaria

13 Department of Medical Oncology and Clinical Pharmacology B, National Institute of Oncology Országos Onkológiai Intézet, Budapest Ráth György u. 7-9, Budapest 1122, Hungary

14 Global Clinical Operations, Mylan Healthcare GmbH, Freundallee 9A, 30173 Hannover, Germany

15 Global Product Safety and Risk Management, Mylan, Building 4 Trident Place, Mosquito Way, Hatfield AL10 9UL, UK

16 Product Safety and Risk Management, Mylan, 781 Chestnut Ridge Road, Morgantown, WV 26505, USA

17 Clinical Development, Biocon Research Ltd, 131, Jigani Road, RK Twp, Bommasandra Industrial Area, Bangalore, Karnataka 560099, India 